\title{
Destabilization of fast particle stabilized sawteeth in ASDEX Upgrade with electron cyclotron current drive
}

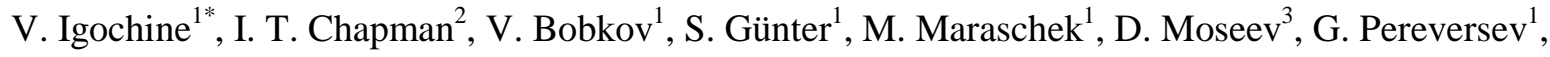 \\ M. Reich ${ }^{1}$, J. Stober ${ }^{1}$ and ASDEX Upgrade team ${ }^{1}$ \\ ${ }^{1}$ MPI für Plasmaphysik, Euratom-Association, D-85748 Garching, Germany \\ ${ }^{2}$ EURATOM/CCFE Association, Culham Science Centre, Abingdon, Oxfordshire OXI4 3DB, UK \\ ${ }^{3}$ EURATOM-Risф DTU, Risф National Laboratory for Sustainable Energy, Technical University of \\ Denmark, DK-4000 Roskilde, Denmark
}

\begin{abstract}
It is often observed that large sawteeth trigger the neoclassical tearing mode (NTM) well below the usual threshold for this instability. At the same time, fast particles in the plasma core stabilize sawteeth and provide these large crashes. The paper presents results of first experiments in ASDEX Upgrade for destabilization of fast particle stabilized sawteeth with electron cyclotron current drive (ECCD). It is shown that moderate ECCD from a single gyrotron is able to destabilize the fast particle stabilized sawteeth. A reduction in sawtooth period by about $40 \%$ was achieved in first experiments. These results show that ECCD can be used as a tool for control of sawteeth also in presence of fast particles.
\end{abstract}

\section{Introduction}

In magnetically confined fusion plasmas, a variety of magnetohydrodynamic (MHD) instabilities can occur, driven by gradients of kinetic pressure or current density. The sawtooth oscillation is one of the fundamental instabilities in tokamaks. It is associated with abrupt changes in central plasma confinement due to growth of an $(m, n)=(1,1)$ mode, where $m$ and $n$ are poloidal and toroidal mode numbers [1]. Whilst the plasma usually survives the drops in core temperature and density due to this instability, the triggering of other, more dangerous instabilities is the main concern. It is often observed that large sawteeth trigger the neoclassical tearing mode (NTM) well below the threshold for this instability [2]. Example of NTM triggered by big sawtooth crash in ASDEX Upgrade is shown in figure 1. It is also shown that changes of the ion cyclotron resonance heating (ICRH) power change the sawtooth period [3]. In future reactors like ITER, the fusion born

\footnotetext{
*email: valentin.igochine@ipp.mpg.de
} 
$\alpha$-particles will stabilize the $(1,1)$ mode and lead to larger sawteeth which are more probable to trigger NTMs (see discussion about NTM triggering in Ref[4]). Thus, avoidance of large sawteeth is of prime importance for a robust scenario in ITER. The second possible problem for ITER could come from redistribution of the fast particles during the crash. Such redistribution is observed with collective Thomson scattering (CTS) diagnostic in TEXTOR [5].
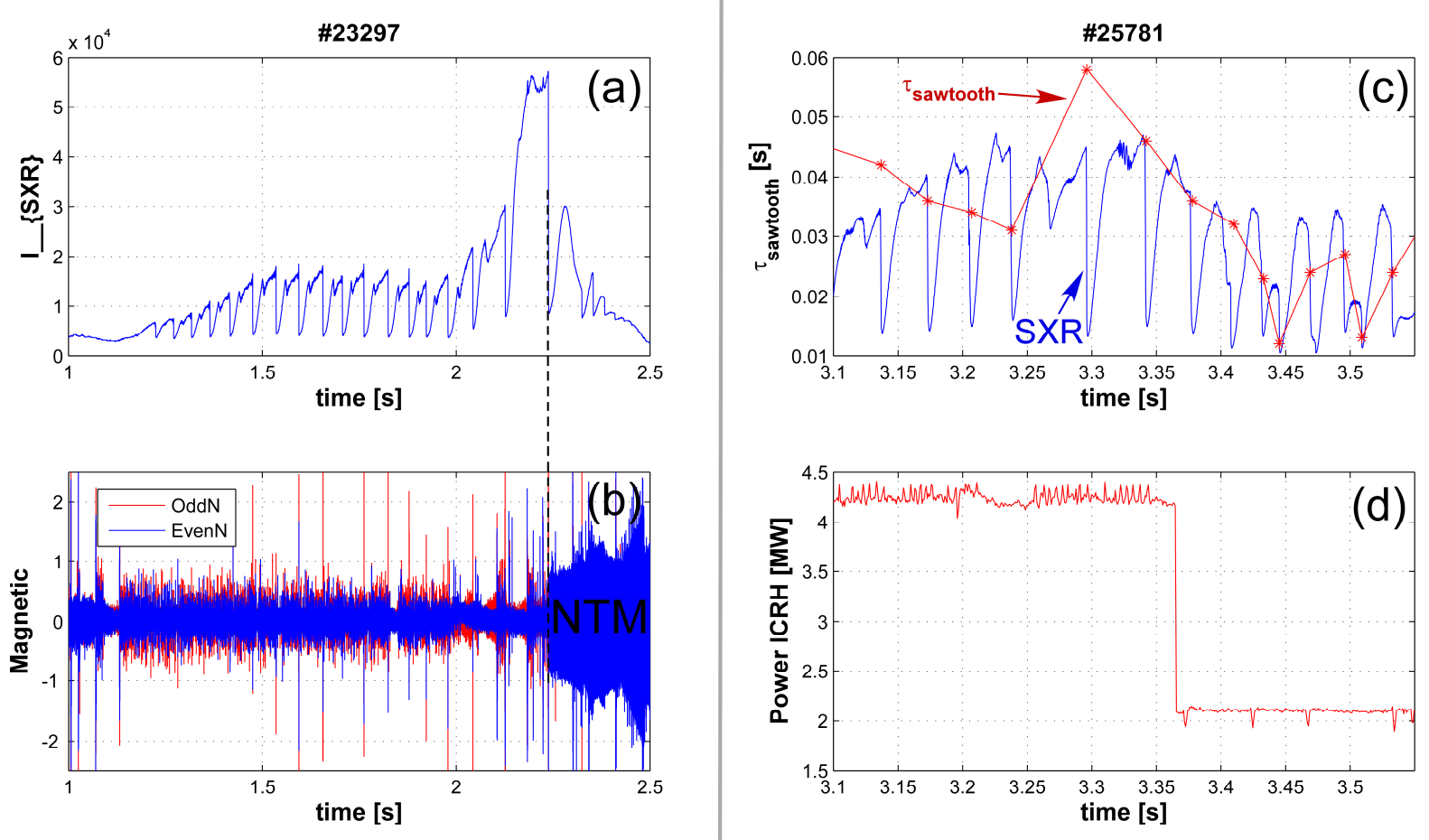

Figure 1. (Color online) Triggering of NTM by large sawtooth crash is shown for ASDEX Upgrade discharge 23297: (a) central Soft X-ray channel; (b) magnetic signals. Changes of the sawtooth period (c) with changes of applied ICRH power (d) are shown. The average sawtooth period is reduced with reduction of ICRH heating.

The main aim of this work is reproducing an ITER relevant situation. The fast $\alpha$-particles are imitated with central ICRH heating and Neutral Beam Injection (NBI) in ASDEX Upgrade. This heating produces a population of very energetic ions in the plasma core which stabilizes the $(1,1)$ mode and increases the sawteeth period [6]. The fast particle distribution is peaked in the plasma centre (inside $\mathrm{q}=1$ surface) for central ICRH heated discharges in ASDEX Upgrade [7]. The fast particles with energies higher than $1 \mathrm{MeV}$ were also observed by fast particle detector in ICRH heated discharges.

The destabilization of the sawteeth is achieved by local changes of the current profile with electron cyclotron current drive (ECCD). This is achieved by changing the ECRH mirror angle. (Previous experiments in ASDEX Upgrade used variation of toroidal magnetic field to change ECCD 
deposition position, which is not possible in ITER [8,9].) In this paper we report the first results which were recently achieved on ASDEX Upgrade.

\section{Sawteeth destabilization experiments}

The reported experiments employ the high $q_{95} \mathrm{H}$-mode parameters of ASDEX Upgrade tokamak: $I_{\text {plasma }}=1 M A, n_{e}=8 \cdot 10^{19} \mathrm{~m}^{-3}, B_{\text {toroidal }}=2.5 T, q_{95}=4.7$. The heating scheme includes high ICRH power $\left(P_{I C R H}=4.3-4.4 M W\right)$ and one NBI source $\left(P_{N I}=2.5 M W\right)$. Toroidal magnetic field and plasma current are constant during the experimental phase. The $140 \mathrm{GHz}$ ECRH system is used to heat the plasma $\left(P_{E C R H}=0.8 M W\right)$ in the core. This allows gaining two different goals simultaneously:

- Influence of the ECRH heating on the ECCD experimental results is strongly diminished since core electron heating remains relatively constant during the scan.

- The impurity accumulation is reduced which provides stable discharge conditions [10].

The temperature profiles are almost identical with and without applied ECCD [11]. Thus, effect of pure heating from ECCD gyrotron is negligible.

Central ICRH heating produces a population of fast ions in the plasma core which stabilize the $(1,1)$ mode and increase the sawtooth period. The destabilization of the sawteeth is achieved by local changes of the current profile with electron cyclotron current drive (ECCD). The radial position of the ECCD is swept poloidally during the discharge, corresponding to a sweep of the resonant location from inside to outside of the $\mathrm{q}=1$ surface $\left(P_{E C C D, \text { gyrotron }}=1.0 \mathrm{MW}\right)$. Changes of the sawtooth period depending on time and on the deposition position are shown for the co-current drive case in figure 2. The experiment shows that co-current ECCD inside the $\mathrm{q}=1$ surface destabilizes sawteeth and reduces the sawtooth period by about $40 \%$. Consequently, the outside deposition stabilizes sawteeth. The deposition positions and deposition profiles for different time points are shown in figures $3(\mathrm{a}, \mathrm{b})$. The width of the deposition profile is relatively broad in comparison with previous experiments [8]. On the one hand, this is unfavourable for sawtooth control but on the other hand, a similar situation is expected in ITER. This broad deposition profile is a result of the off-axis injection geometry and has pure geometrical origin. The advantage of the present system is an almost selfsimilar deposition profile (see figure $3 \mathrm{~b}$ ). Influence of the applied ECCD on equilibrium profiles was modelled with transport code ASTRA [12]. The result show small changes of safety factor profile with strong changes of shear in the vicinity of $q=1$ surface (see figure $3 \mathrm{c}$ ). The total electron cyclotron driven current varies from $1.9 \mathrm{kA}$ to $2.5 \mathrm{kA}$ which is about $1 \%$ of the total current inside 
$\mathrm{q}=1$ radius. Our results confirm recent findings from ToreSupra that moderate ECCD is able to destabilize fast particle stabilized sawteeth [13].
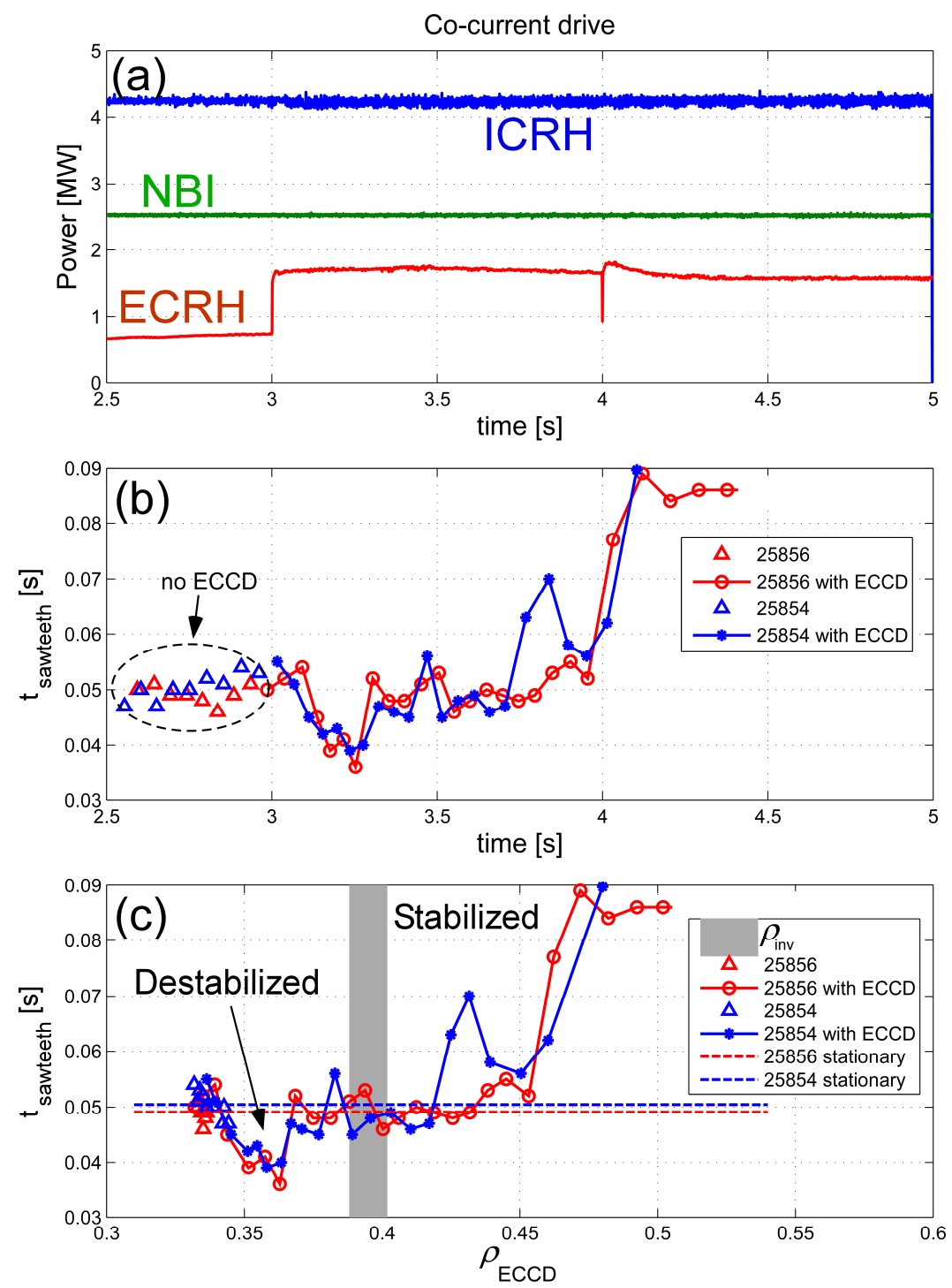

Figure 2. (Color online) Heating powers during the experiments are shown in the upper figure 2(a). The central ECRH heating is always on. Co-current ECCD is applied from 3 second. Changes of the sawteeth period depending on time (2b) and maximum of ECCD deposition position (2c) are shown for co-current drive case. The current drive position changes from inside of the $q=1$ surface to the outside as shown in second figure. The sawtooth inversion radius is marked. 

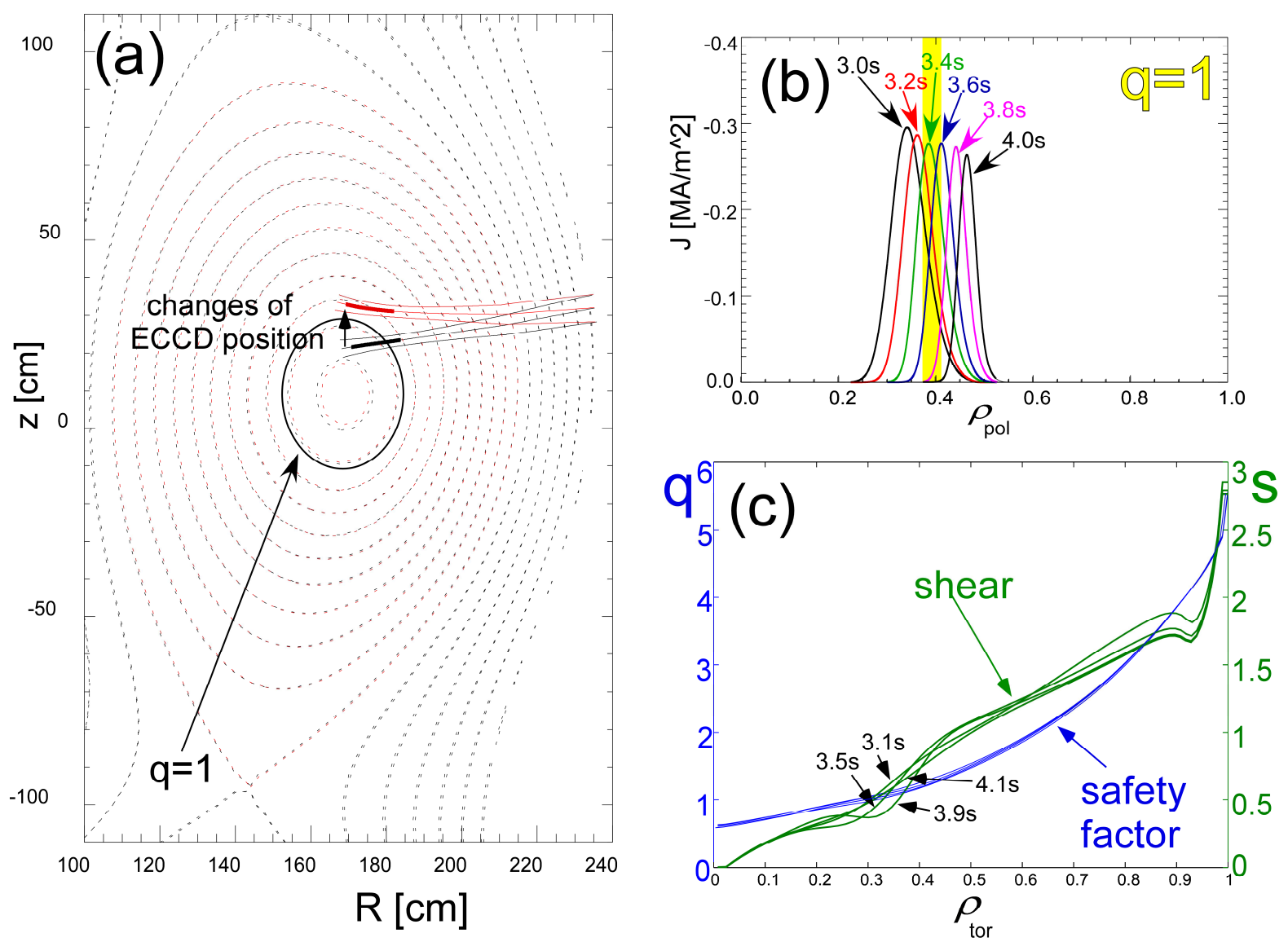

Figure 3. (Color online) Discharge 25856: (a) changes of the co-current ECCD locations during the scan from inside to the outside of $q=1$ surface; (b) co-ECCD deposition profiles for different times; (c) changes of safety factor profile and shear profile.

\section{Stability analysis of co-current drive experiments}

Simulations of shot 25856 have been compared to the Porcelli linear crash trigger criterion from $[14,15]$. This analysis results from linear modelling thus it cannot be used to correctly describe the sawtooth period but does provide, however, an indication of the stability of the plasma to the internal kink which is thought to be responsible at least for the onset of the sawtooth crash (see review in Ref.[16]). By using the Hagis Monte-Carlo drift-kinetic code the contribution of the energetic ions to the potential energy of the internal kink mode can be numerically assessed to evaluate the efficacy of varying the magnetic shear to control sawtooth in the presence of fast ion stabilizing effects. Consequently, the closer the total $\delta \mathrm{W}$ is to the threshold predicted for onset of the sawtooth crash, the lower the sawtooth period is likely to be. The linear modelling is performed using the Mishka-1 linear MHD stability code [17] to evaluate the fluid drive for the kink mode and the Hagis waveparticle interaction code [18] to evaluate the change in the potential energy of the kink mode due to the presence of fast ions. 
The effects of the fast ions are taken into account by making approximations for both the NBI and ICRH populations. The ICRH distribution function is assumed to be bi-Maxwellian in form, as in references $[19,20]$ :

$$
f_{h}^{I C R H}=\left(\frac{m}{2 \pi}\right)^{3 / 2} \frac{n_{c}(r)}{T_{\perp}(r) T_{\|}^{\frac{1}{2}}(r)} \exp \left(-\frac{\mu B_{c}}{T_{\perp}(r)}-\frac{\left|E-\mu B_{c}\right|}{T_{\|}(r)}\right),
$$

where the particle energy $E=m v^{2} / 2$, the magnetic moment $\mu=m v_{\perp}^{2} / B, \|$ and $\perp$ represent the components parallel and perpendicular to the magnetic field respectively, $B_{c}$ is the critical field strength at the resonance and $n_{c}$ is the local density evaluated at $B=B_{c}$. The passing NBI ions can be approximately represented by a non-symmetric distribution which is slowing down with respect to energy and Gaussian with respect to radius and pitch angle [21], such that

$$
f_{h}^{p}=C \exp \left(\frac{\psi-\psi_{0}}{\Delta \psi}\right) \exp \left(\frac{\lambda-\lambda_{0}}{\Delta \lambda}\right) \frac{1}{E^{3 / 2}-E_{c}^{3 / 2}} \operatorname{Erf}_{c}\left[\frac{E-E_{0}}{\Delta E}\right],
$$

where $\Delta \psi=0.1, \lambda_{0}=0.5, E_{0}=80 \mathrm{keV}, E_{c}=3 \mathrm{keV}, \Delta E=3.5 \mathrm{keV}$. The effect of the ECCD is taken into account by perturbing the current profile used as input to the HELENA equilibrium code [22] by the non-inductive driven currents predicted by TORBEAM. In each of the three generated equilibria, the radius of the $\mathrm{q}=1$ surface is held fixed, as observed on the soft X-ray measurements, and accordingly the safety factor on-axis is scaled slightly (less than 10\%). These equilibria represent three different time points of the same discharge with different position of the ECCD deposition. The change in the magnetic shear resulting from the ECCD is the major determining factor influencing the stability of the kink mode with respect to the linear crash trigger thresholds.

Figure 4 shows the change in the potential energy of the internal kink mode resulting from the fluid drive together with that arising from the presence of ICRH and NBI fast ions, all at three different times during discharge 25856 (as illustrated in figure 4). At $t=3.25 ; 3.5 ; 3.75 \mathrm{~s}$, the peak of the deposition of the ECCD predicted by TORBEAM is at $\rho_{\text {pol }}=0.367,0.398,0.438$ respectively. The contribution of the ICRH and NBI fast ions does not change during this time since the $q=1$ surface is assumed not to move in accordance with experimental observation. However, since the magnetic shear changes significantly at the $q=1$ surface, the total normalized change in the potential energy of the mode is strongly affected, where $\delta \hat{W}_{t o t} \approx \delta W_{t o t} / s_{1}$, where $s_{1}=2 V_{q=1}\left(\frac{d q}{d V}\right)_{q=1}$ is shear at $\mathrm{q}=1$ surface. At the earliest time, when the peak of the ECCD is inside the measured inverison radius, the increase in $s_{1}$ results in an enhanced destabilisation, manifest as 
$\delta \hat{W}_{\text {tot }}$ being close to the predicted Porcelli crash trigger threshold. However, as the ECCD is scanned outwards and moves to approximately at $q=1(\mathrm{t}=3.5 \mathrm{~s})$ and outside $q=1(\mathrm{t}=3.75 \mathrm{~s})$, the shear decreases and so the internal kink is stabilised, in qualitative agreement with the increase in the sawtooth period observed in the experiment.

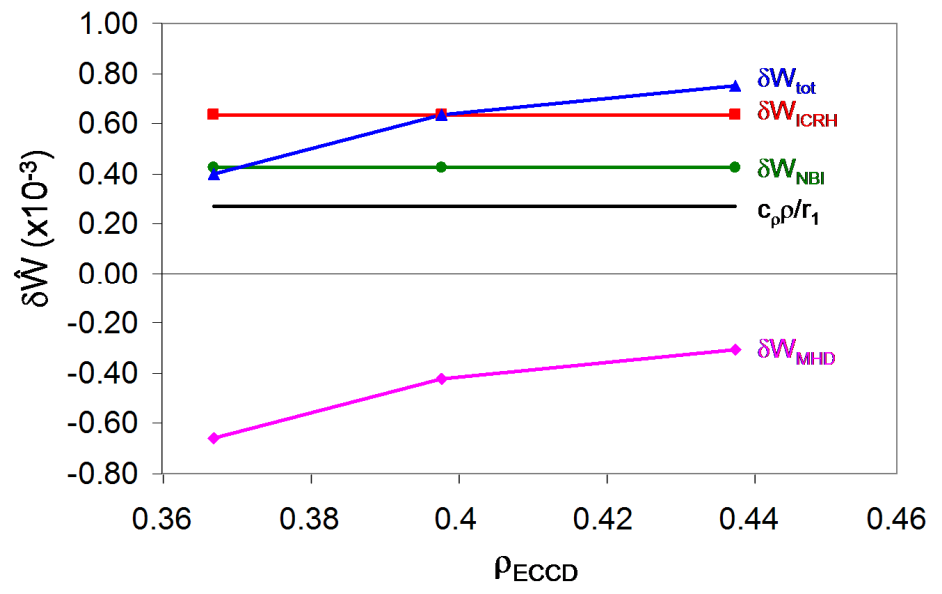

Figure 4. (Color online) The change in the potential energy of the internal kink mode resulting from the fluid drive ( $\left.\delta W_{M H D}\right)$, the stabilizing effect of the ICRH ( $\left.\delta W_{I C R H}\right)$ and ( $\left.\delta W_{N B I}\right)$ fast ions, and the total mode energy ( $\delta W_{t o t}$ ) all shown for three different locations of the ECCD deposition at $\mathbf{t}=\mathbf{3 . 2 5}, 3.5,3.75 \mathrm{~s}$. Also shown for comparison is the threshold level $\left(c_{p} \rho / r_{1}\right.$, Porcelli criteria) required for a sawtooth crash to be triggered according to the model in reference [14]

\section{Conclusions}

Large sawteeth produced by a combination of ICRH and NBI heating in ASDEX Upgrade are able to trigger NTMs. First experiments in ASDEX Upgrade show that moderate ECCD from a single 1MW gyrotron is able to destabilize the fast particle stabilized sawteeth. The reduction in sawtooth period by about $40 \%$ was achieved with co-current drive deposited inside the $\mathrm{q}=1$ radius. The stabilization influence of ICRH on sawteeth is seen by variation of ICRH power in the experiment. First modelling results also suggest a strong stabilization influence from ICRH produce fast population. We diminish influence of pure ECRH heating on our results by applying additional central ECRH heating. Thus, the reported results reflect essentially influence of the current drive on sawteeth.

The predicted sawtooth amplitude in ITER is about the critical size to trigger NTM [23]. Thus, $40 \%$ reduction of the sawtooth period could be sufficient to avoid such triggering. At the same time, further optimization is necessary if our estimations for ITER are wrong. We think that further 
strong reduction of the sawtooth period could be achieved by increase of the current drive and optimization of current drive position.

It is clear that further experiments are necessary to explore the case of several gyrotrons which will be installed on ASDEX Upgrade with identical mirror systems. It is planned that CTS diagnostic will provide distribution function in new experiments which is necessary for more accurate modelling of fast particle influence on the sawteeth.

\section{Acknowledgments}

VI gratefully acknowledges useful discussions with Prof. Hartmut Zohm about sawtooth physics. VI is grateful to Dr. Manuel Garcia-Munoz for useful remarks about fast particles. IC: This work was partially funded by Research Councils UK under grant EP/G 003955.

\section{References}

[1] Hastie R.J., Astrophysics and Space Science 256, p.177 (1998)

[2] O. Sauter, E. Westerhof, M. Mayoral et al., Phys. Rev. Lett. 88, 105001 (2002)

[3] J.P. Graves, et.al. , Nuclear Fusion 50 (2010) 052002 (6pp)

[4] I.T. Chapman, et.al., Nucl. Fusion 50 (2010) 102001

[5] S.K. Nilsen, et.al., Plasma Phys. Control. Fusion 52 (2010) 092001

[6] I.T. Chapman, et.al., Plasma Phys. Control. Fusion 49 (2007) B385

[7] K. Sassenberg, et.al., Plasma Phys. Control. Fusion 51 (2009) 065003

[8] A. Manini, et.al., 32nd EPS Conference on Plasma Phys. Tarragona, 27 June - 1 July 2005 ECA Vol.29C, P-4.073 (2005)

[9] A. Mück, et.al., Plasma Phys. Control. Fusion 47 (2005) 1633-1655

[10] R.Neu et.al., Plasma Phys. Control. Fusion 44 (2002) 811

[11] F. Ryter et.al., Nucl. Fusion 43 (2003) 1396

[12] G.V. Pereversev, P.N. Yushmanov, “ASTRA Automated System for Transport Analysis", IPP report, IPP 5/98 , Feb. 2002

[13] M. Lennholm, et.al., Phys. Rev. Lett. 102, 115004 (2009)

[14] Porcelli F, Boucher D and Rosenbluth M 1996 Plasma Phys. Control. Fusion 382163

[15] Sauter O et al 1998 Theory of Fusion Plasmas, Proc Joint Varenna-Lausanne International Workshop, Varenna (AIP) p403

[16] I.T. Chapman., Plasma Phys. Control. Fusion 53 (2011) 013001 (36pp) 
[17] A.B. Mikhailovskii, G.T.A. Huysmans, S.E. Sharapov and W.O. Kerner, Plasma Phys. Rep. 23844 (1997)

[18] S.D. Pinches et al, Comput. Phys. Commun., 111, 133 (1998) Release Version 8.09

[19] Graves JP, CooperWA, Coda S, Eriksson LG and Johnson T 2006 Theory of Fusion Plasmas, Joint Varenna-Lausanne International Workshop p350

[20] Dendy RO et al 1995 Phys. Plasmas 21623

[21] Chapman IT et al 2009 Nucl. Fusion 49035006

[22] G.T.A. Huysmans, J.P. Goedbloed and W. Kerner, Proceedings of the CP90 Conference on Computer Physics, (World Scientific, Singapore, 1991), p. 371

[23] T.C. Hender, et.al., Nucl. Fusion 47 (2007) S128-S202 